Check for updates

Cite this: Chem. Commun., 2020 56, 13033

Received 29th July 2020

Accepted 21st September 2020

DOI: $10.1039 / \mathrm{d} 0 \mathrm{cc} 05172 \mathrm{a}$

rsc.li/chemcomm

\section{Catechols: a new class of carbonic anhydrase inhibitors $\dagger$}

\author{
Katia D'Ambrosio, (D)*a Simone Carradori, (D) ${ }^{\mathrm{b}}$ Stefania Cesa, (D) ${ }^{\mathrm{c}}$ Andrea Angeli, ${ }^{d}$ \\ Simona M. Monti, ${ }^{a}$ Claudiu T. Supuran (D) ${ }^{d}$ and Giuseppina De Simone (D) *a
}

To date, catechols have been only poorly investigated as carbonic anhydrase (CA) inhibitors. Here we report the first structural information on the CA inhibition mechanism of these molecules, showing that they adopt a peculiar binding mode to the enzyme active site which involves the zinc-bound water molecule and the "deep water".

Human CAs (hCAs) are zinc containing enzymes, which catalyze the inter-conversion of carbon dioxide into bicarbonate ions and protons. $^{1,2}$ Fifteen different isoforms have been identified so far, among which only twelve are catalytically active (CA I-IV, VA-VB, VI-VII, IX, and XII-XIV). ${ }^{1}$ These enzymes present a common fold, consisting of a central $\beta$-sheet, surrounded by helical connections and additional $\beta$-strands, with an active site located in a deep and wide cavity, spanning from the protein surface to the center of the molecule. The $\mathrm{Zn}^{2+}$ ion, which is essential for catalysis, is located at the bottom of this cavity and is coordinated in a tetrahedral geometry by three conserved histidine residues and a water molecule/hydroxide ion. ${ }^{1,3}$ The latter is in turn involved in a network of hydrogen bonds, which plays a key role in the catalytic mechanism (Fig. S1, ESI $\dagger$ ). Indeed, it interacts with the hydroxyl moiety of the conserved Thr199 residue and with two water molecules, located on two opposite sides of the cavity: the first one, named the "deep water", is located in a hydrophobic pocket, while the second one is in a hydrophilic environment toward the entrance of the active site. ${ }^{1}$

Since the carbon dioxide hydration reaction is of essential importance in a variety of physiological processes based on ion

\footnotetext{
${ }^{a}$ Istituto di Biostrutture e Bioimmagini-CNR, Naples, Italy.

E-mail: katia.dambrosio@cnr.it, giuseppina.desimone@cnr.it;

Tel: +39-081-2532044, +39-081-2534579

${ }^{b}$ Department of Pharmacy, "G. d'Annunzio" University of Chieti-Pescara, 66100 Chieti, Italy

${ }^{c}$ Dipartimento di Chimica e Tecnologie del Farmaco, Sapienza University of Rome, 00185 Rome, Italy

${ }^{d}$ Neurofarba Department, Università degli Studi di Firenze, Sesto Fiorentino, Florence, Italy

$\dagger$ Electronic supplementary information (ESI) available: Experimental details, Fig. S1-S5 and Tables S1, S2. See DOI: 10.1039/d0cc05172a
}

transport, gas exchange and pH balance, abnormal levels and/ or activities of hCAs have been associated with several diseases, which include glaucoma, metabolic syndrome, epilepsy and cancer. ${ }^{1}$ Consequently, these enzymes in the last few years have been taken into account as targets to design inhibitors for biomedical applications. ${ }^{1}$

CA inhibitors (CAIs) can be grouped into two main classes: the inhibitors that bind to the active site coordinating the catalytic zinc ion, such as primary sulfonamides and their isosteres (sulfamates and sulfamides), ${ }^{4}$ dithiocarbamates, ${ }^{5}$ and xanthates, ${ }^{6}$ and the inhibitors that bind to the active site without interacting with the metal ion. ${ }^{1}$ Molecules belonging to this second class can either be anchored to the zinc-bound water molecule (ZBW), as reported for phenols ${ }^{7,8}$ and polyamines, ${ }^{9}$ or bound in another region of the active site cavity, as observed for coumarins ${ }^{10,11}$ and lacosamide. ${ }^{12}$ Interestingly, some inhibitors like carboxylic acids can show both the binding modes, depending on their chemical structure. ${ }^{13-17}$

Recently, great interest has been shown on natural products as a source of lead compounds for developing CAIs. ${ }^{18,19}$ Indeed, many advantages can be associated with the utilization of natural products in drug discovery, such as their structural and chemical diversity unmatched by any synthetic chemical collection and their capability to interact with biological targets. ${ }^{20}$ In this context, several phenolic compounds among secondary plant metabolites were identified as inhibitors of the hCA activity. ${ }^{18,19,21-23}$ Among these compounds there is chlorogenic acid (CGA) (compound 1 in Fig. 1 ), which shows $K_{\mathrm{I}}$ values against several hCA isoforms varying from a high micromolar to a nanomolar range (see Table $\mathrm{S} 1, \mathrm{ESI} \dagger){ }^{23}$

CGA is a biologically active dietary catechol, playing several therapeutic roles. ${ }^{24}$ It contains two functional groups capable of interacting with the CA active site, namely the carboxylic and the hydroxyl moieties. Thus, with the aim to clarify the interaction mechanism of this compound with CAs, we undertook a crystallographic investigation of the hCA II/1 adduct. hCA II was chosen as a model isoform for crystallization, since it readily forms 
<smiles>O=C(/C=C/c1ccc(O)c(O)c1)OC1CC(O)(C(=O)O)C[C@H](O)[C@H]1O</smiles>

1<smiles>Oc1cccc(O)c1</smiles><smiles>Oc1ccc(O)cc1</smiles>

4
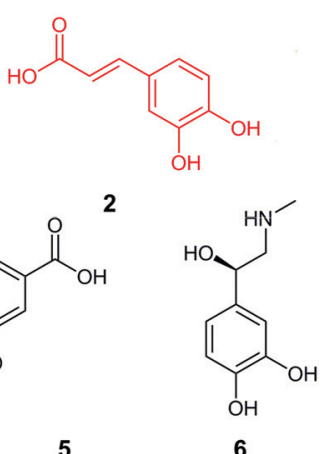

Fig. 1 Chemical structures of the compounds 1-6.

crystals and many studies have been reported on its adducts with all classes of known inhibitors. ${ }^{1}$

Crystals of the hCA II/1 complex were obtained by soaking hCA II native crystals, grown as previously described, ${ }^{17,25}$ in a saturated solution of the inhibitor for 24 hours. The structure was analyzed by difference Fourier methods using the native hCA II coordinates ${ }^{3}$ as a reference, and subsequently refined with the CNS program ${ }^{26,27}$ to crystallographic $R_{\text {work }}$ and $R_{\text {free }}$ values of $0.159 / 0.192$ in the $25.0-1.60 \AA$ resolution range. The statistics for data collection and refinement are reported in Table S2 of the ESI. $\dagger$ After the first stages of refinement, inspection of the $|F \mathrm{O}-F \mathrm{c}|$ and $|2 F \mathrm{O}-F \mathrm{c}|$ electron density maps revealed the presence of a molecule within an enzyme active site. However, the shape of the density was not compatible with the compound CGA, but well matched with its hydrolysis product caffeic acid (CFA) (compound 2 in Fig. 1) (Fig. 2), which was then added to the crystallographic coordinates and

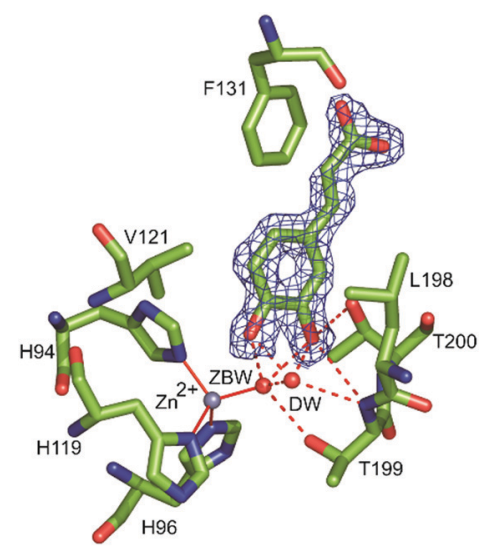

Fig. 2 Active site region of the complex obtained by soaking the hCA II crystals in a CGA solution. The $\sigma A$-weighted $|2 F o-F c|$ map (contoured at $1.0 \sigma$ ) relative to the inhibitor molecule is also shown together with the newly formed CFA, which perfectly fits this density, the zinc ion and the residues involved in inhibitor recognition. Continuous lines indicate the zinc ion coordination, whereas the dashed lines indicate the hydrogen bond distances. Water molecules are indicated as red circles. DW indicates the "Deep Water". The zinc bound solvent molecule was assumed to be a water molecule in agreement with the hCA II neutron structure solved at $\mathrm{pH} 9.45{ }^{28}$ refined as described in ESI. $\dagger$ Further support to this finding came from the observation that when the hCA II crystals were soaked in a solution containing CFA and the structure of the corresponding adduct was determined, the electron density maps in the enzyme active site were perfectly superimposable to those obtained when hCA II was co-crystallized with CGA (Fig. S2, ESI †).

To explain why under our experimental conditions CGA was hydrolyzed to CFA, several hypotheses could be advanced: (i) the hydrolysis was a consequence of the basic features of the crystallization buffer; (ii) it was due to the esterase activity of the enzyme; and (iii) it was mediated by the enzyme but only in the presence of the crystallization buffer. Thus, additional experiments were carried out to clarify this point.

First, CGA was incubated for $24 \mathrm{~h}$ with the crystallization buffer and analyzed by HPLC at different times (Fig. S3 and S4, ESI $\dagger$ ). The experiment clearly showed that CGA does not undergo hydrolysis to CFA, if maintained up to $24 \mathrm{~h}$ under the described conditions. Rather, it slowly and only partially evolves into an unknown species, whose retention time $\left(t_{\mathrm{R}}\right)$ is lower than that of CGA. From the analysis of the spectral data, this species could be postulated to be the 1-caffeoylquinic acid formed upon the acyl migration of CGA (3-caffeoylquinic acid). Anyway, the recovery yield for CGA at the end of the experiment was $84 \pm 1 \%$, in agreement with previously reported studies, showing that most of the hydrolytic procedures involving CGA are based on harsh alkaline conditions $(2-4 \mathrm{~N} \mathrm{NaOH}$ at $25-30{ }^{\circ} \mathrm{C}$ from $30 \mathrm{~min}$ to $6 \mathrm{~h}$ ). ${ }^{29}$

Secondly, CGA was incubated with the enzyme under the same conditions of the inhibition assays (see the ESI $\dagger$ ) and again analyzed by HPLC at different times (Fig. S5, ESI $\dagger$ ). Also in this case, CGA did not undergo hydrolysis to CFA.

Finally, the inhibition assays of hCA II with CGA and CFA were carried out at different incubation times of the enzyme/ inhibitor adduct (i.e. $15 \mathrm{~min}, 3 \mathrm{~h}, 6 \mathrm{~h}$ and $24 \mathrm{~h}$ ) (Table 1).

Table 1 Inhibition of hCA II with CGA, CFA and the standard AAZ at different incubation times

\begin{tabular}{lc}
\hline Compound & $K_{\mathrm{I}}^{a}(\mu \mathrm{M})$ \\
\hline Incubation time $15 \mathrm{~min}$ & 30.1 \\
CGA & 1.61 \\
CFA & 0.012 \\
AAZ & \\
Incubation time $3 \mathrm{~h}$ & 30.1 \\
CGA & 1.61 \\
CFA & 0.012 \\
AAZ & \\
Incubation time $6 \mathrm{~h}$ & 30.1 \\
CGA & 1.61 \\
CFA & 0.012 \\
AAZ & \\
Incubation time $24 \mathrm{~h}$ & 25.6 \\
CGA & 1.61 \\
CFA & 0.012 \\
AAZ &
\end{tabular}

${ }^{a}$ Mean from 3 different assays, by a stopped flow technique (errors were in the range of $5-10 \%$ of the reported values). 
A

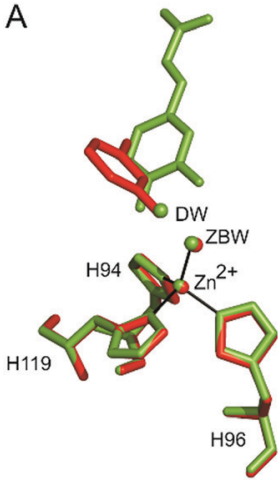

Fig. 3 Structural superposition of CFA (green) with (A) compound $\mathbf{3}^{16}$ (red) and (B) compound $\mathbf{5}^{31}$ (orange) when bound to the hCA II active site. Zinc ion coordination and the "deep water" are also shown.

The results reported in Table 1 clearly show that $K_{\mathrm{I}} \mathrm{S}$ of the two catechols are diverse from each other and do not change with time, thus excluding the fact that under the experimental conditions used for the inhibition assays, CGA could be hydrolyzed to CFA by the enzyme. These data were in agreement with the HPLC analysis.

Altogether these three experiments clearly demonstrate that GCA is hydrolyzed to CFA only in the simultaneous presence of hCA II and a crystallization buffer and under physiological conditions this molecule does not act as a suicide inhibitor, as instead reported for other CAIs such as coumarins ${ }^{10,11}$ and sulfocoumarins. ${ }^{30}$ Nevertheless the unexpected formation of CFA under our experimental conditions provided very interesting perspectives on the design of new CAIs. Indeed, the binding mode of this molecule to the CA active site is very peculiar and has never been observed for other phenol and polyphenol CAIs. In detail, the inhibitor is anchored to the enzyme by means of the two hydroxyl groups (catechol) which are hydrogen bonded both to the ZBW and to the "deep water" (Fig. 2). One of these hydroxyls is also at hydrogen bond distances from the Thr200OG1 and Thr199N atoms. The organic scaffold of the inhibitor establishes several hydrophobic interactions (distance < 4.0 Å) with residues Val121, Phe131 and Leu198, whereas the carboxylate functionality points towards the protein surface and does not interact with any protein residue.

Interestingly, the comparison of the CFA binding mode with that of the other phenols and/or polyhydroxy phenols not containing adjacent hydroxyl groups (see compounds $\mathbf{3}$ and $\mathbf{4}$ in Fig. 1$)^{16}$ reveals substantial differences. Indeed, although the latter molecules still bind the ZBW of the enzyme through a hydrogen bond, they do not interact with the "deep water", which is displaced by one of their hydroxyl groups. Also, the position of the aromatic ring of these inhibitors is completely different with respect to that of CFA (Fig. 3A).

It is worth noting that the binding mode of CFA has been so far observed only for some carboxylic acids. ${ }^{16,31}$ In the latter cases, the two oxygens of the carboxylate group are nearly in the same position of the two catechol hydroxyl groups (Fig. 3B). Even more interesting is that all the known CAIs containing both phenolic and carboxylic functionalities bind to the CA enzyme either within the active site anchoring their carboxylic moiety to the $\mathrm{ZBW},{ }^{16,32}$ or on the border of the active site occluding it, as observed for the coumarin hydrolysis products. ${ }^{10,11}$ In the case of CFA, the presence of the catechol moiety allows the accomplishment of a different binding mode, which involves both the ZBW and "deep water".

To date, there has been only another catechol whose structure in complex with a CA isoform has been determined, namely L-adrenaline (compound 6 in Fig. 1), which has been described as a CA activator. ${ }^{33}$ In this case, the molecule binds in a completely different region of the active site, probably due to its different charge, which is affected by the $\mathrm{pH}$ of the crystallization buffer. Indeed, at the $\mathrm{pH}$ used in the crystallization experiments, L-adrenaline should be positively charged due to the presence of the amine functionality, whereas CFA should be negative.

In conclusion, in this paper due to the unexpected formation of CFA at the catalytic site, we provided the first structural information on the binding mode of cathecols to CAs. Due to the peculiar features of this binding, catechols emerge as a new class of CAIs, which are worth exploring further.

This work was supported by a grant from the MIUR-PON "Ricerca e Innovazione" 2014-2020 (grant MOLIM ONCOBRAIN LAB) and a grant from the Regione Campania PO FESR 20142020 (grant eMORFORAD).

\section{Conflicts of interest}

There are no conflicts to declare.

\section{Notes and references}

1 V. Alterio, A. Di Fiore, K. D'Ambrosio, C. T. Supuran and G. De Simone, Chem. Rev., 2012, 112, 4421-4468.

2 J. E. Coleman, Ann. N. Y. Acad. Sci., 1984, 429, 26-48.

3 A. E. Eriksson, T. A. Jones and A. Liljas, Proteins, 1988, 4, 274-282.

4 D. Neri and C. T. Supuran, Nat. Rev. Drug Discovery, 2011, 10, 767-777.

5 F. Carta, M. Aggarwal, A. Maresca, A. Scozzafava, R. McKenna and C. T. Supuran, Chem. Commun., 2012, 48, 1868-1870.

6 F. Carta, A. Akdemir, A. Scozzafava, E. Masini and C. T. Supuran, J. Med. Chem., 2013, 56, 4691-4700.

7 S. K. Nair, P. A. Ludwig and D. W. Christianson, J. Am. Chem. Soc., 1994, 116, 3659-3660.

8 R. A. Davis, A. Hofmann, A. Osman, R. A. Hall, F. A. Muhlschlegel, D. Vullo, A. Innocenti, C. T. Supuran and S. A. Poulsen, J. Med. Chem., 2011, 54, 1682-1692.

9 F. Carta, C. Temperini, A. Innocenti, A. Scozzafava, K. Kaila and C. T. Supuran, J. Med. Chem., 2010, 53, 5511-5522.

10 A. Maresca, C. Temperini, L. Pochet, B. Masereel, A. Scozzafava and C. T. Supuran, J. Med. Chem., 2010, 53, 335-344.

11 A. Maresca, C. Temperini, H. Vu, N. B. Pham, S. A. Poulsen, A. Scozzafava, R. J. Quinn and C. T. Supuran, J. Am. Chem. Soc., 2009, 131, 3057-3062.

12 C. Temperini, A. Innocenti, A. Scozzafava, S. Parkkila and C. T. Supuran, J. Med. Chem., 2010, 53, 850-854.

13 I. Bertini, C. Luchinat and A. Scozzafava, Biochim. Biophys. Acta, 1976, 452, 239-244.

14 I. Bertini, C. Luchinat and A. Scozzafava, Bioinorg. Chem., 1978, 9, 93-100. 
15 K. Hakansson, M. Carlsson, L. A. Svensson and A. Liljas, J. Mol. Biol., 1992, 227, 1192-1204.

16 D. P. Martin and S. M. Cohen, Chem. Commun., 2012, 48, 5259-5261.

17 E. Langella, K. D'Ambrosio, M. D'Ascenzio, S. Carradori, S. M. Monti, C. T. Supuran and G. De Simone, Chemistry, 2016, 22, 97-100.

18 A. Karioti, F. Carta and C. T. Supuran, Molecules, 2016, 21, 1649.

19 A. Karioti, F. Carta and C. T. Supuran, Curr. Pharm. Des., 2016, 22, 1570-1591.

20 G. D. Wright, Microb. Biotechnol., 2019, 12, 55-57.

21 I. Gulcin, A. Scozzafava, C. T. Supuran, H. Akincioglu, Z. Koksal, F. Turkan and S. Alwasel, J. Enzyme Inhib. Med. Chem., 2016, 31, 1095-1101.

22 I. Gulcin, A. Scozzafava, C. T. Supuran, Z. Koksal, F. Turkan, S. Cetinkaya, Z. Bingol, Z. Huyut and S. H. Alwasel, J. Enzyme Inhib. Med. Chem., 2016, 31, 1698-1702.

23 A. Mollica, M. Locatelli, G. Macedonio, S. Carradori, A. P. Sobolev, R. F. De Salvador, S. M. Monti, M. Buonanno, G. Zengin, A. Angeli and C. T. Supuran, J. Enzyme Inhib. Med. Chem., 2016, 31, 1-6.

24 M. Naveed, V. Hejazi, M. Abbas, A. A. Kamboh, G. J. Khan, M. Shumzaid, F. Ahmad, D. Babazadeh, X. FangFang, F. ModarresiGhazani, L. WenHua and Z. XiaoHui, Biomed. Pharmacother., 2018, 97, 67-74.
25 K. D'Ambrosio, S. Carradori, S. M. Monti, M. Buonanno, D. Secci, D. Vullo, C. T. Supuran and G. De Simone, Chem. Commun., 2015, 51, 302-305.

26 A. T. Brunger, Nat. Protoc., 2007, 2, 2728-2733.

27 A. T. Brunger, P. D. Adams, G. M. Clore, W. L. DeLano, P. Gros, R. W. Grosse-Kunstleve, J. S. Jiang, J. Kuszewski, M. Nilges, N. S. Pannu, R. J. Read, L. M. Rice, T. Simonson and G. L. Warren, Acta Crystallogr., Sect. D: Biol. Crystallogr., 1998, 54, 905-921.

28 S. Z. Fisher, A. Y. Kovalevsky, J. F. Domsic, M. Mustyakimov, R. McKenna, D. N. Silverman and P. A. Langan, Biochemistry, 2010, 49, 415-421.

29 M. Nardini, E. Cirillo, F. Natella, D. Mencarelli, A. Comisso and C. Scaccini, Food Chem., 2002, 79, 6.

30 K. Tars, D. Vullo, A. Kazaks, J. Leitans, A. Lends, A. Grandane, R. Zalubovskis, A. Scozzafava and C. T. Supuran, J. Med. Chem., 2013, 56, 293-300.

31 L. A. Woods, O. Dolezal, B. Ren, J. H. Ryan, T. S. Peat and S. A. Poulsen, J. Med. Chem., 2016, 59, 2192-2204.

32 C. L. Lomelino and R. McKenna, Acta Crystallogr., Sect. F: Struct. Biol. Commun., 2019, 75, 166-170.

33 C. Temperini, A. Innocenti, A. Scozzafava, A. Mastrolorenzo and C. T. Supuran, Bioorg. Med. Chem. Lett., 2007, 17, 628-635. 